\title{
Archäologische Artefakte mit griechischem Psalm 90 in apotropäischer Funktion
}

\section{Hinführung und Problemaufriss}

In Europa ist Ray Lewis nahezu unbekannt, in den USA eine Legende. Jedenfalls ist er das für alle jene, die sich für American Football begeistern. Seine Lebensgeschichte hat alles, was eine mitreißende Verfilmung verlangen würde: Hoch talentiert und entschlossen, zwischenzeitlich unter Mordanklage, geläutert und bekehrt, zweimaliger Super Bowl-Sieger, Mentor und Idol junger Spieler und Abtritt von der großen Bühne des Profi-Sports am Tag seines größten Erfolgs. ${ }^{1}$ Am dritten Februar 2013 endet seine siebzehnjährige Karriere. Siebzehn Jahre spielte er immer nur für den einen Club, seine Baltimore Ravens, und immer nur auf derselben Defensivposition des Middle Linbackers. An jenem dritten Februar lief er in den Mercedes-Benz-Superdome in New Orleans ein, mit goldenen Stollenschuhen: Außen im Zehenbereich war in stahlblau die Aufschrift ,Psalms 91' zu lesen. ${ }^{2}$ Nach dem Sieg gegen die San Francisco 49ers in einem engen, mitreißenden Match läuft er eine Ehrenrunde und gibt Interviews. Er trägt ein schwarzes T-Shirt mit goldenen Lettern darauf, wieder mit ,Psalms 91“. Dieses T-Shirt trug er bereits bei seinem allerletzten Heimspiel in Baltimore, wo es daraufhin in den Medien Aufmerksamkeit erregte. In Blogs und Kommentaren fragten sich viele, was es mit diesem Psalm auf sich hat und was Lewis damit mitzuteilen gedenkt. Entsprechend gingen Los Angeles Times und New York Times im Januar 2013 auf Shirt und Botschaft ein, verwiesen gar kurz auf Inhalt und Aussage des Psalms und druckten diesen zudem ab, damit sich die Leserschaft selbst ein Bild davon machen konnte. ${ }^{3}$ Lewis selbst äußerte sich zu diesem Psalm wie folgt: ${ }^{4}$

For me, through the ups and downs, the roller coasters of 17 years, you have to find a safe place [...]. You have to find that place that is very quiet in your head, and anytime I read it, anytime I come across it, my Bible, the first Scripture in there is Psalms 91.

\footnotetext{
1 Dies lässt sich sicherlich so behaupten, obgleich er bereits 2001 in Tampa den Super Bowl XXXV gewann.

2 Einen Tag vor dem Endspiel findet sich eine enthusiastische Beschreibung der Sportschuhe im Internet von Juska 2013.

3 Vgl. Schilken 2013 mit einer englischen Fassung des Psalms; Mills 2013. Die Popularität und das Interesse an diesem Psalm vor allem in den Vereinigten Staaten von Amerika belegen belletristisch und plakativ ausgerichtete Veröffentlichungen wie z. B. Ruth 2007, deren Buch auch als „Military Edition“, „for Youth“ und „Workbook“ erhältlich ist; Copeland 2012; Maples 2014.

4 Mills 2013.

D Open Access. (C) 2018 Thomas J. Kraus, publiziert von De Gruyter. (c) BY-NC-ND Dieses Werk ist lizenziert unter der Creative Commons Attribution-NonCommercial-NoDerivatives 4.0 Lizenz.

https://doi.org/10.1515/9783110619928-006
} 
Dieser „sichere Ort“, den Lewis in Psalm $91 \mathrm{zu}$ finden glaubt, wurde dadurch auch noch all jenen Menschen zugänglich gemacht, die allenfalls von den Aufdrucken „Psalms 91“ gehört oder diese auf Fotos gesehen hatten und damit nicht wirklich etwas anfangen konnten. Denn bereits die bloße Präsenz und Sichtbarkeit der Aufdrucke auf den Schuhen und dem T-Shirt - zigmal live verbreitet über Fernsehkanäle und die Anzeigetafeln im Stadion - konnte von jenen gedeutet und in ihrer Aussage identifiziert werden, die wussten, um was es sich bei „Psalms 91“ - möglicherweise sogar in Bezug auf Ray Lewis - handelte. Neben diesen Insidern, sogenannten Wissenden um und über Psalm 91, waren dann also auch die eigentlichen Outsider und Interessierten durch die Medien in Kenntnis gesetzt.

Ortswechsel, Zeitreise: Russland in den Wirren der Oktoberrevolution und des anschließenden Bürgerkriegs, irgendwann zwischen 1917 und 1920. Der Arzt Juri Shiwago trifft mit einer Gruppe Rotarmisten auf eine Einheit junger Weißarmisten. Das Scharmützel ist nur kurz und für die Kadetten verheerend. Shiwago fällt die Aufgabe $\mathrm{zu}$, nach Verwundeten zu suchen, um diese zu verarzten. Zweimal findet Shiwago ein Amulett mit einem Stück Papier, auf dem sich ebenjener Psalm 91 findet. Den Telefonisten der Roten konnte das Amulett in kirchenslawischer Fassung nicht retten, doch ein Kadett der Weißen überlebt, weil die Kugel durch das Amulett in russischer Sprache abgehalten wurde. Boris Pasternaks Erzähler erläutert die Szene um Psalm 91 näher, dessen Beliebtheit und Gebrauch in der breiten Bevölkerung sowie dessen angenommene Schutzfunktion, auch dessen Unterschiede in der von Shiwago gefundenen russischen und kirchenslawischen Fassung. Unsichtbar, unter der Uniform getragen, ist das Amulett stets dort präsent, wo sich auch der Besitzer befindet. Seine Bedeutung erschließt der Autor seiner Leserschaft durch einen Erzähler, so dass auch der Nichteingeweihte versteht, was hier vor sich gegangen sein mag. ${ }^{5}$

Diese beiden Episoden aus der Welt des Sports und der Weltliteratur verdeutlichen bereits das Wesentliche und die Zielsetzung der vorliegenden Darstellungen:

a) Die Beliebtheit und Bezeugung des Psalms (als Psalm 91 im hebräischen, sogenannten masoretischen Text gezählt [= fortan Psalm 91 ${ }^{\mathrm{MT}}$ ] und vor allem dann als Psalm 90 in der griechischen Übersetzung der Septuaginta [= fortan als Psalm $\left.90^{\mathrm{Lxx}}\right]$ ) in apotropäischer Funktion in der Spätantike wird im Überblick dargeboten. Denn insgesamt hat sich zu diesen beiden Beispielen nichts Wesentliches zwischen damals und heute verändert.

b) Gleichzeitig kommt es dadurch zu einem Nachweis von Psalm $90^{\mathrm{LXX}}$ als populärstem und am besten bezeugten Text der Bibel.

c) (a) und (b) äußern sich in unterschiedlicher Form und Materialität, wodurch sich wiederum bestimmte Verwendungs- und Anwendungszwecke/-bereiche ergeben.

d) Die Sichtbarkeit und Unsichtbarkeit sowie die Präsenz von Text (und natürlich zugeordneten ikonographischen, symbolischen, zeichenhaften u. a. Elementen)

5 Hierzu Pasternak 1992, 458-459. 
spielen eine besondere Rolle für die angenommene Wirksamkeit, das Verständnis und die Vorstellung bei den Menschen der (späten) Antike wie auch heute noch.

e) Gerade (d) erfährt anhand von zwei ausgewählten Beispielen aus bestimmten Kategorien archäologischer Objekte mit Psalm 90 ${ }^{\mathrm{Lxx}}$ bzw. Versen oder einzelnen Worten aus diesem eine ausführliche Demonstration.

\section{Griechischer Psalm 90 in apotropäischer Funktion im Überblick}

Werden Menschen nach den bekanntesten und populärsten Bibeltexten gefragt, so nennen die meisten - sofern sie überhaupt etwas mit der Bibel in Verbindung bringen können - meist und sofort das Vaterunser, die Zehn Gebote, das Schma Jisrael (Deuteronomium 6,4-9), Psalm 23 („Der Herr ist mein Hirte“), Johannes 3,16 („Denn Gott liebte die Welt so sehr [...]”), irgendetwas aus der Bergpredigt oder aus der Offenbarung bzw. Apokalypse des Johannes. Dass aber ein einzelner Psalm, sowohl in seiner hebräischen Form (Psalm 91 ${ }^{\mathrm{MT}}$ ) und noch vielmehr in der griechischen der durch archäologische Artefakte am besten bezeugte und populärste Bibeltext ist, wird viele überraschen. Vollständig oder nur in einzelnen Versen bzw. einfach den Anfangsworten findet sich der Psalm auf Wandinschriften, in Gräbern und Grabkammern, auf Medaillons und Anhängern, Armbändern, Ringen, Holztäfelchen, Sarkophagen und Papyrus- und Pergamentstücken. ${ }^{6}$ Die überwiegende Mehrheit dieser Objekte stammt aus der Spätantike, mit einer momentan für das vierte bis sechste Jahrhundert zu veranschlagenden Präferenz. Diese Popularität und die konkrete Verwendung für Übelabwehr und Schutz gegen alle Arten von Bösem war bereits für den hebräischen Psalm vorgezeichnet, denkt man an die Miniaturrolle 11Q11 aus Qumran mit drei ansonsten unbekannten Hymnen und einer Fassung des Psalms. Andere archäologische Zeugnisse (z. B. magische Schalen/Zauberschalen und Fragmente aus der Kairoer Genizah) oder aber auch konkrete Ausweise für die entsprechende apotropäische Verwendung des Psalms im Talmud, den Targumim und Midraschim und etwa dem Sefer Shimmush Tehillim aus dem elften Jahrhundert, Dem Buch des magischen Gebrauchs der Psalmen, das sicher auf einer langen Vorgeschichte und alte Traditionen beruht, zeigen, dass auch das Judentum dem apotropäischen Gebrauch des Psalms aufgeschlossen gegenüberstand. ${ }^{7}$ So belegt die rabbinische Tradition, dass der Psalm - zusammen mit anderen Texten - bei Prozessionen gesungen wurde und

6 Vgl. beispielsweise den Überblick an Objekten in Kraus 2005, 39-73. Ferner zu Armbändern Kraus 2005/2006, 114-27 und Tafeln 2-3 und zu einzelnen Objekten aus den genannten Kategorien Kraus 2014, 825-838.

7 Vgl. Rebiger 2003. 
sogar offiziell rezitiert werden sollte. ${ }^{8}$ Demnach überrascht es nicht, dass auch die Christen der ersten Jahrhunderte diese Konventionen und Rituale fortführten, vielmehr noch erweiterten und entsprechend ihrer sozio-kulturellen Umgebung und Beeinflussung anpassten. Denn auch inhaltlich war der Psalm bestens geeignet vor allen Arten von Übel zu bewahren. ${ }^{9}$ Begünstigend wirkt sich aus, dass zwei Verse des Psalms auch im Neuen Testament zitiert werden, nämlich im Kontext der Versuchungsgeschichte Jesu durch den Diabolos, der selbst letztendlich mit Hilfe eines Schriftzitats Jesus endlich überreden will, seiner Versuchung nachzugeben (Lukas 4,10-11; Matthäus 4,6). Ergo weckte der Psalm bei den frühchristlichen Schriftstellern alsbald Aufmerksamkeit, sowohl in deren Kommentierungen zu den Evangelien als auch nochmals in Kommentaren zu den Psalmen. ${ }^{10}$ Damit ist die Beliebtheit und Verwendung des Psalms für diverse Zwecke vorprogrammiert, insbesondere für apotropäische Zwecke, da der Psalm inhaltlich eben genau diese Dimensionen und den Hoffnungsaspekt ausdrückt. Darüber trifft das alles in den für den vorliegenden Kontext besonders relevanten Gebieten Ägyptens, Syriens und Palästinas, aber auch in anderen Grenzregionen des römischen Reichs auf eine Welt voller diverser und diffus erscheinender religiöser Vorstellungen, in die gerade auch die apotropäische Funktion des Psalms sehr gut passt. ${ }^{11}$ Leider wird das auch heute noch nur selten innerhalb der Theologie wahrgenommen und wenn, dann allenfalls als spezielles und besonderes Phänomen, ${ }^{12}$ dessen theologische und sozio-kulturelle Komponente jedoch kaum Berücksichtigung findet. Womöglich rührt dies daher, dass unter Theologen ein gewisses Unbehagen gegen alle Formen von - auch offizieller - Magie und Synkretismus vorherrscht. ${ }^{13}$

\section{Typologie der archäologische Objekte mit Psalm $90^{\mathrm{Lxx}}$}

Natürlich ist die vor Jahren von mir erstellte, private und unveröffentlichte Database von archäologischen Objekten mit Psalm $90^{\mathrm{Lxx}}$ mittlerweile überholt, erscheinen immer neue Editionen weiterer Zeugnisse mit diesem Psalm bzw. gelangen mir immer wieder weitere Informationen über Objekte zur Kenntnis. ${ }^{14}$ Darüber hinaus wird zu

8 So etwa als Lied für die Geschlagenen (yShab 6,2,8b), Lied gegen die Dämonen (yEr 10,11,26c) und Lied gegen Plagen(geister) (bSchevu 15b).

9 Vgl. Kraus 2005, 42-45.

10 Vgl. Kraus 2005, 42-45; Breed 2014, 298-302.

11 So kürzlich auch Breed 2014, 302-303.

12 Vgl. Sanzo 2014, 106-120 für Ps $90^{\mathrm{LXx}}$.

13 Vgl. Kraus 2014, 826-827.

14 Vgl. Kraus 2005, 47-59. 
klären sein, wie mit Artefakten mit Text in anderen Sprachen (z. B. Syrisch, Koptisch und Lateinisch) zu verfahren sein wird. ${ }^{15}$ Dennoch bedarf auch ein vorläufiger und unvollständiger Datenbestand einer gewissen Strukturierung, die durch die folgende vorläufige Typologisierung erreicht wird:Auf Manuskripten ${ }^{16}$ findet sich der gesamte Psalm oder nur Verse aus diesem, teilweise auch mit anderen Texten kompiliert. Die Popularität des Psalms ist durch seine relativ verlässliche Bezeugung auf Handschriftenfragmenten belegt. ${ }^{17}$ Oftmals wurde das Papyrusstück mehrfach gefaltet, so dass ein kleines Päckchen entstand, das in einer Kapsel oder einem anderen Behältnis mitgeführt und/oder umgehängt werden konnte. Entsprechend verhält es sich mit P.Duk.inv. 778, das entsprechend der Angaben der Herausgeber achtmal vertikal und einmal horizontal gefaltet war und auf einer Seite das Vaterunser mit Doxologie und auf der anderen unseren Psalm und die Überschrift des folgenden Psalms im Buch der Psalmen bewahrt. Auf beiden Seiten finden sich noch Kreuze, so dass die christliche Zuordnung gesichert ist. Das kleine Päckchen konnte damit problemlos mitgeführt werden, war also stets präsent, und deshalb wirksam, um vor Unheil zu schützen. ${ }^{18}$ Diese apotropäische Funktion hing aber nicht von der Sichtbarkeit und Identifizierbarkeit des Psalms selbst ab. Sicherlich war die Kapsel oder das Medaillon für andere sichtbar, der konkrete Inhalt jedoch nicht. Allerdings dürfte auch die Unsichtbarkeit keine wirkliche Rolle für die angenommene Wirksamkeit gespielt haben, diente das Behältnis dazu, das Papyrusstück zu schützen und gleichzeitig leichter mitführen zu können.

Einen weiteren Typus stellen die sogenannten Bovৎ-Täfelchen dar. ${ }^{19}$ Von den bekannten Täfelchen mit der Buchstabenfolge $\beta o v \varsigma$ weisen drei den ägyptischen

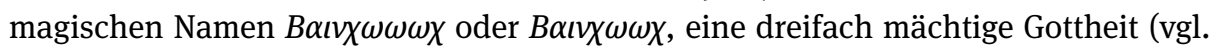
Pistis Sophia 137 und 147), drei weitere den Anfang von Psalm 90 ${ }^{\mathrm{LXX}}$ auf. Der wirkliche konkrete Zweck dieser Täfelchen ist nach wie vor eine Angelegenheit für Spekulationen. So wurde $\beta o v \varsigma$ mit der Dekangottheit $B \omega \varsigma$ gleichgesetzt, was aber aus linguistischen Überlegungen heraus problematisch erscheint. ${ }^{20}$ Dienten die Tafeln

15 Vgl. Kraus 2009, 139 und 140 Anm. 14. Zudem stellt ein großes Problem dar, dass manche Objekte sich heute - vermutlich - in Gebieten befinden, für die der Zutritt aufgrund gewalttätiger Konflikte unmöglich scheint, bzw. dass Objekte eben so auch zerstört werden oder verloren gehen.

16 Wie in der Papyrologie üblich findet der Begriff ,Papyrus‘ auch für Manuskripte/Fragmente Verwendung, die aus anderem Material sind (z. B. Pergament, Holz, Blei oder Silber), da eine entsprechende Inventarisierung in Sammlungen mit ,P.‘ (= Papyrus) stattfindet. Vgl. Kraus 2007, 13-24.

17 Kraus 2005, 49-52, verweist auf einundzwanzig Papyri. Ferner La'da/Papathomas 2004, 107-113; Kraus 2013, 148-153; Sanzo 2014, 106-120.

18 Vgl. La'da/Papathomas 2004, 93-113; Kraus 2006a, 243-245; Kraus 2013, 148-149.

19 Vgl. Nachtergael, 2002, 93-101; Kraus 2008, 479-491.

20 An dieser Stelle danke ich Joachim Friedrich Quack, Ägyptologisches Institut, Heidelberg, sehr für die Diskussionen und die Zusendung der relevanten Abschnitte seiner noch unveröffentlichten Habilitationsarbeit. Offensichtlich ist die Reduktion von $\beta$ ouৎ zu $\beta \omega \varsigma$ oder $\beta$ o $~ n i c h t$ belegt. 
zum Schutz gegen ebensolche bösen Mächte oder sollten die angeführten Namen und Worte den Schutz vor allem Übel unterstützen oder sogar herbeiführen? Oder steckt gleich ganz etwas anderes hinter diesen Täfelchen?

Es überrascht keineswegs, wenn sich gerade Psalmenverse auf Türstürzen finden. Vor allem Psalm 120,8 ${ }^{\mathrm{LXX}}$ war ganz besonders prädestiniert für diese Verwendung. ${ }^{21}$ Doch auch Psalm $90^{\mathrm{LXx}}$ mit seinen Wünschen nach Geleit, Schutz und Sicherheit war als Text ebenso geeignet. Die meisten der hier relevanten Türstürze wurden in Syrien entdeckt. Sie sind alle auf Griechisch beschriftet, stammen von verschiedenen Gebäuden und bewahrten neben Textlichem auch Kreuze, ein Rad oder eine sonnenähnliche Form und andere nicht-textliche Elemente. Natürlich waren die textlichen und bildhaften Elemente auf den Türstürzen für alle sichtbar angebracht, ihre Präsenz sollte das Haus und diejenigen, die da ein- und ausgingen, beschützen, was durch die Segenssprüche explizit zum Ausdruck kommt. ${ }^{22}$

In Grabkammern verhält sich die Angelegenheit etwas anders. Natürlich ist der Schutz- und Hoffnungscharakter des Psalms auch hier bestens geeignet. Allerdings stellt sich die Frage, an wen sich letztlich die Botschaft des Psalms dann richtet und in welcher Art und Weise dieser wirksam sein soll. In der Nekropole von Gabbari im Westen Alexandrias, die auch von Christen im vierten bis sechsten Jahrhundert genutzt wurde, befindet sich eine Grabkammer mit verschiedenen Inschriften in rotbräunlicher Tinte auf Stein. Diese Inschriften stehen an Wänden, Decken und in den loculi, den Grabkammern bzw. Grabnischen selbst. Eine dieser Nischen weist Psalm 90,1 $\mathrm{b}^{\mathrm{LXX}}$ zusammen mit Kreuzen und Akklamationen auf. Ein weiteres Zeugnis für einen solchen Gebrauch des Psalms ist eine Grabanlage in Kertsch auf der Krim in Südrussland, die wahrscheinlich heute nicht mehr existiert bzw. nunmehr unauffindbar ist. ${ }^{23}$ Das relevante Grab wird auf das Jahr 491 datiert und beinhaltete viele loculi. In der Grabkammer selbst sowie den Nischen waren diverse Texte und Kreuze in roter Farbe aufgetragen. In einer Nische wurde der vollständige Psalm $90^{\mathrm{LXX}}$ - noch dazu in einer mit heutigen kritischen Ausgaben übereinstimmenden Fassung - geschrieben und zwar in der Art, dass die erste Zeile auf der linken Wandseite begann, über die hintere und rechte Seite fortgeführt wurde und erst dann auf der linken Seite in Zeile zwei der Text fortgesetzt wurde. Es scheint die Inschrift, also Psalm 90 ${ }^{\mathrm{LXx}}$, den Verstorbenen umgeben zu haben. Der Psalm mag hier, noch mehr als in der Grabkammer in Gabbari, den Toten schützend umfassen. Bei beiden Gräbern allerdings ist nach deren Verschluss für den Vorbeikommenden nichts von den Texten und weiteren Elementen sowie deren beabsichtigte Wirkung zu erkennen. Die Inschriften richten sich demnach an die Verstorbenen innerhalb der Gräber, vielleicht auch als apotropäische

21 Vgl. Kraus 2006b, 58-75.

22 Vgl. Kraus 2009, 140; Kraus 2014, 837.

23 Ich danke Antonio Enrico Felle, Università degli Studi di Bari, Dipartimento di Scienze dell‘Antichità e del Tardo Antico, für Hinweise auf diese Inschrift. Vgl. auch Felle 2006, 206-209 (Nr. 438-443). 
Macht für die Reise in das Jenseits oder Fortleben in einer anderen Welt, möglicherweise auch für die Zeit bis hin zur Auferstehung. ${ }^{24}$ Sei das, wie es sei, die Präsenz der Inschrift ist entscheidend für deren unterstellte Wirksamkeit. Die Sichtbarkeit bzw. Unsichtbarkeit unterscheidet zwischen Verstorbenen und/oder allen Arten von Übeln auf der einen und den Vorbeikommenden und Außenstehenden auf der anderen Seite. $^{25}$

Zwei Sarkophage aus Me'eleyya/Meğeleyya (Apamene) im oberen Syrien bewahrten den Anfang von Psalm 90 ${ }^{\mathrm{LXX}}$ außen auf einer Längsseite. IGLS IV $1488^{26}$ ist vollständig erhalten und trägt eine vierzeilige Inschrift, die sich aus typischen Formeln und Phrasen zusammensetzt:

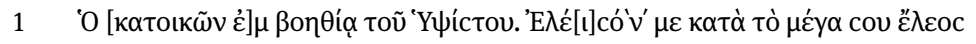

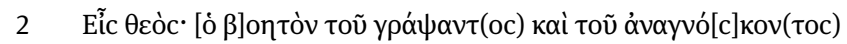

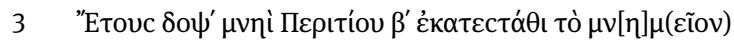

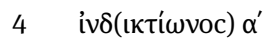

Wer in der Hilfe des Höchsten wohnt. Erlöse mich gemäß deiner großen Gnade.

[Es gibt nur] einen Gott, der dem hilft, der schrieb und der das liest.

Im Jahr 774, dem zweiten Peritios, wurde das Grab errichtet

in der ersten Indiktion.

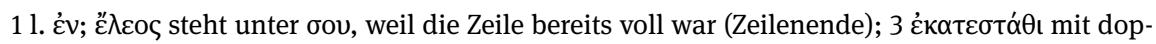

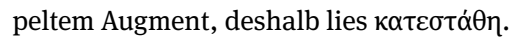

Neben dem Anfang des Psalms kommt die weit verbreitete und populäre Eĩ̧ $\theta \varepsilon o ́ \varsigma-$ Formel, d. h. die monotheistische „Ein-Gott-Formel“, in Anwendung sowie eine typische Wendung für die Angabe des Datums, die dann das Jahr 463 n. Chr. darstellt. Von besonderem Interesse ist aber die direkte Anrede von Schreiber und Leser des Texts, die unter Gottes Schutz (näher attribuiert als der „eine Gott“) stehen mögen. Dieser Segenswunsch mag sich auch auf die verstorbene Person beziehen, die im Sarkophag beigesetzt ist. Allenfalls implizit könnte die Inschrift eine Warnung für diejenigen darstellen, die im Begriff sind, den Sarkophag zu öffnen und zu plündern. Der „eine Gott“ ist hier zuständig, Sarkophag und auch Verstorbener stehen unter seinem Schutz. Die Inschrift weist auch eine Art Rad in der Mitte der Längsseite auf, deren acht Speichen ein Kreuz repräsentieren könnten. Wie dem auch sei, das Symbol - sei es Rad, Kreuz

24 Weitere Informationen zu den Inschriften besonders bei Felle 2006, 206-210 (Nr. 438-445). Ferner Kraus 2013, 146-147; Kraus 2014, 834-836.

$25 \mathrm{Zu}$ nennen sei hier beispielsweise noch eine Inschrift in einer Kellernische in Antinoopolis (elSheikh Ibada) in roter Farbe mit Psalm 90,11-13 und 120,7-8, einem Ensemble, das sich auch in Kertsch findet. Vgl. Felle 2006, 59-60 (Nr. 35).

26 Eine Zeichnung bietet Prentice 1908, 184 (Nr. 207). 
oder Sonne - unterstützt die schützende und segnende Wirkung der Inschrift. Und auch die fragmentarische, vierzeilige Inschrift des zweiten Sarkophags, IGLS IV 1483

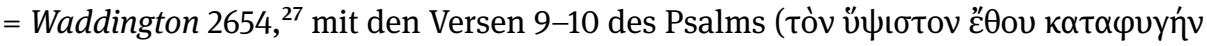

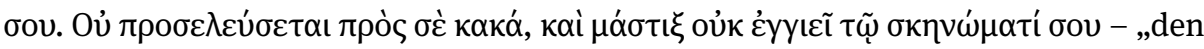
Höchsten hast du zu deiner Zuflucht gemacht. Zu dir wird Böses nicht hinkommen, und eine Geißel wird deinem Zelt nicht nahe kommen“), verweist zusammen mit dem Kreuzzeichen deutlich auf einen Segensspruch gegen böse Mächte und alles Übel, fungiert demnach wiederum apotropäisch. ${ }^{28}$ Beide Sarkophage stellen also eine deutlich andere Ausrichtung hinsichtlich Sichtbarkeit und Präsenz des Textes (und des Symbols) dar als etwa die Inschriften in den beiden Grabkammern, da auch der Vorbeikommende sehen und lesen konnte, was das Grabmal als Ausstattung (nach außen hin) aufwies, und so angesprochen und womöglich ermahnt wurde.

Dass dann auch der Psalm im Umfeld von Kirchen bzw. Kapellen zu finden ist, liegt auf der Hand. Das Beispiel einer fragmentarischen Inschrift aus dem antiken Petra kann dies vor Augen führen. Aus der Umgebung einer Kirche und einer Begräbnisstätte stammen Fragmente einer Wandbeschriftung in roter Farbe auf Stein. Das meiste des Psalms $90^{\mathrm{Lxx}}$ kann auf den Steinfragmenten rekonstruiert werden. Wahrscheinlich stammen die Bruchstücke aus dem pastophorion einer Kirche, d. h. dem Arbeitsraum eines Priesters, der neben dem Heiligtum angebaut oder von diesem mit einer Mauer abgetrennt wurde. Aber warum sollte ein Priester, noch dazu in einer solchen Umgebung, besonderen Schutzes gegen Übel bedürfen? Handelt es sich ganz einfach um den für den Priester explizit zugesicherten Schutz Gottes, eben durch die sichtbare Präsenz des Texts dem Priester damit in diesem Raum stets in Erinnerung gerufen? Jedenfalls ist die Omnipräsenz böser Mächte in den Vorstellungen der Menschen der (Spät-)Antike gesichert, die auch hier mit hineinspielen könnte. ${ }^{29}$

In meiner Datenbank finden sich auch fünf Ringe mit den Eingangsworten von Psalm 90 ${ }^{\mathrm{LXx}}$. Ein Ring ist aus Silber, die anderen aus Bronze. Gerade die kleinen Medaillons der Ringe legen auch den Umfang des belegten Texts fest, so dass meist nur einige wenige Worte des Psalms dort Platz fanden. Nur ein Ring hat auch Text auf dem Band des Rings. Alle diese Ringe sind sichtbare Repräsentationen einer gewissen Einstellung und alle, die mit den Worten bzw. dem Text selbst vertraut waren, konnten die Botschaft dahinter sowie die Funktion des Texts problemlos identifizieren: Alle Formen des Übels sollten abgewehrt, der Träger/die Trägerin des Rings geschützt werden, etwa nach dem Motto ,Ich werde von Gott beschützt, zumindest glaube ich ganz fest daran und beschwöre dies‘. Allerdings sei auch die Frage erlaubt, ob wirklich die Identifizierung der Buchstaben und der Wörter selbst im Vordergrund gestanden hat. ${ }^{30}$ Denn in einer Welt, in der Lesen und Schreiben bei weitem nicht die

27 Vgl. Prentice 1908, 185 (Nr. 208).

28 Hierzu Kraus 2013, 140-142; Kraus 2014, 834.

29 Vgl. Kraus 2014, 836-837.

30 Vgl. Kraus 2014, 837. 
Verbreitung fanden wie gerne heute angenommen, steht sicherlich eher der Glaube an die Wirksamkeit und damit die apotropäische Funktion des Geschriebenen im Vordergrund, ganz gleich, was das nun im Einzelnen war.

\section{Beispiele für Medaillons/Anhänger und Armbänder}

\subsection{Ein Amulett-Anhänger aus dem antiken Kibyra}

Sie sind rund oder mandelförmig, gefertigt aus diversen Materialien, weisen Kombinationen aus Text, ikonographischen, zeichenhaften und symbolischen Elementen auf und scheinen auf einem festen Programm an Motiven und Bildelementen zu basieren. Medaillons und Anhänger sind in jeder Hinsicht faszinierende Repräsentanten von Vorstellungswelten, die sich aus den unterschiedlichsten Gedanken- und Glaubensbereichen zusammensetzen. Viele von ihnen weisen noch Löcher oder Ösen auf, mit Hilfe derer sie an einer Schnur/Kordel mitgeführt bzw. um den Hals gehängt werden konnten. ${ }^{31}$ Auf ihnen finden sich beispielsweise damals äußerst populäre textliche und ikonographische Elemente für apotropäische Objekte: Der Reiterheilige auf dem Pferd mit seiner Lanze im Kampf gegen Repräsentationen des Bösen, der böse Blick (oftmals diagonal durchgestrichen, also entmachtet), die Eĩ̧ $\theta$ có -Formel, das trishagion („Heilig, heilig, heilig“) oder typische emblematische Symbole für die Evangelisten des Neuen Testaments. Ganz gleich, wie die Zusammenstellung letztlich ausfiel, alle diese Medaillons/Anhänger dienten dem einen Zweck: Ihre Träger gegen alle Arten des Übels, gegen Krankheiten und Unglück zu schützen, sie auf sicheren Wegen zu bewahren. ${ }^{32}$

Als Beispiel sei hier ein Amulett vorgestellt, das bei Grabungen im antiken Kibyra (K'́ßupa oder Cibyra Magna, heute Gölhisar, Türkei) entdeckt wurde und vor das sechste Jahrhundert zu datieren ist. Das Objekt ist aus einer Kupferlegierung (Bronze) gefertigt, circa $0,1 \mathrm{~cm}$ dick, $3,5 \mathrm{~cm}$ hoch und 4,3 cm breit. Leider ist nur der obere Teil erhalten (vgl. Abb. 1 und 2). ${ }^{33}$

Möglicherweise gehörte dieses Amulett der Gruppe jener an, die mit Hilfe einer Öse und einer Kordel um den Hals getragen wurden. Generell müssen wir heute mit einer regen Produktionstätigkeit von solchen Amuletten in der (Spät-)Antike rechnen, wie dies steinerne Model für Metallobjekte mit diesem Psalm auch vor Augen führen,

31 Hierzu beispielsweise Foskolou 2014, 334-345.

32 Vgl. Kraus 2013, 142-145; Kraus 2014, 833.

33 Das Amulett wird auch veröffentlicht in der Dissertation von Demirer (Demirer 2013). Eine ausführliche Edition erfolgt durch Demirer/Kraus 2015. 

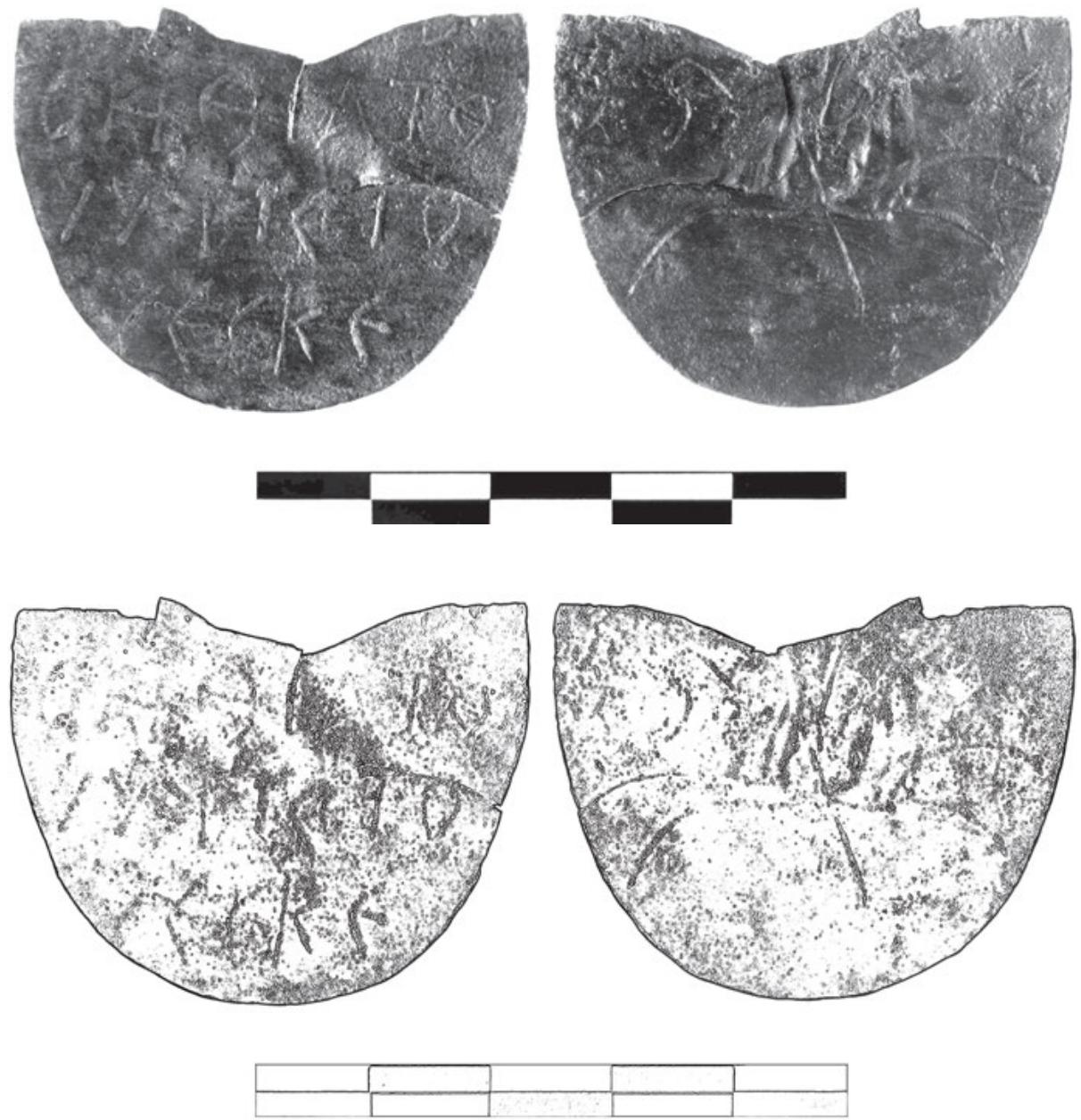

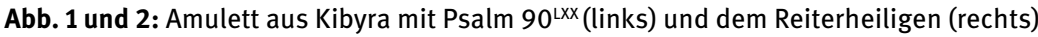

von denen heute immerhin zwei erhalten sind. ${ }^{34}$ Auf der einen Seite sieht man den unteren Teil eines Reiters mit Lanze zu Pferd. Das Pferd selbst ist in Bewegung dargestellt. Entweder bäumt es sich auf oder aber galoppiert es nach rechts. Leider erlaubt der Erhaltungszustand keine Entscheidung darüber, ob wirklich Buchstabenspuren oder Dekorationen auf der linken und rechten Seite des Reiters vorhanden sind. Vom Reiter selbst sind nur mehr sein rechtes Bein und seine Lanze eindeutig zu identifizieren. Das Reiter-Pferd-Ensemble ist durchaus filigran ausgeführt, fein in das Material eingeritzt. Die Gliedmaßen des Pferds erscheinen recht lang, zierlich und geschwungen. Eine Figur liegt unterhalb des Pferds, das von der Lanze bedroht oder sogar

34 Für die beiden Modeln vgl. Foskolou 2014, 336 mit Fig. 5. 
gestochen wird. Wie etliche andere Beispiele zeigen, besteht solch ein Figurenensemble oft aus dem Reiterheiligen (identifiziert mit Salomon oder Sisinnios) mit seiner Lanze im Kampf gegen die Repräsentation des Bösen (dargestellt als Löwe/Löwin mit menschlichem Kopf, als Dämon, auch identifiziert mit Lilith oder Gello/Gyllou). Schon diese eine Seite des Amuletts führt dessen apotropäische Funktion vor Augen. Der Reiter kämpft tatsächlich gegen die real vorgestellte Darstellung des Bösen, dann stellvertretend für die Person, die das Amulett bei sich hat und trägt, und schützt diese. Auf der anderen Seite sind dann noch Reste einer Inschrift in vier Zeilen erhalten, die rasch als Teil von Psalm 90,1LXX erkannt und rekonstruiert werden können:

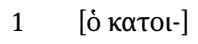

$[\kappa \tilde{\omega} \nu \dot{\varepsilon} v] \beta-$

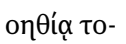

ṽ ن́ฬícto-

$5 v \dot{\varepsilon}<v>c \kappa \dot{\varepsilon}<\pi \eta \kappa \tau \lambda$.>

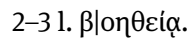

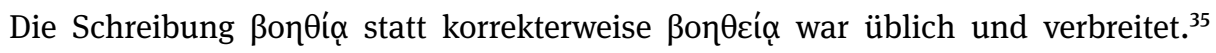

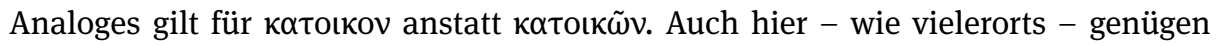
die ersten Worte des Psalms bereits, sei es aus der Überzeugung heraus, dass überhaupt die Macht des Wortes/der Buchstaben hilfreich sei oder aber der ganze Psalm durch dieses pars pro toto bereits in seiner Funktion und Wirkung damit vollständig eingespielt werde. Wie schon erwähnt, erschließt sich die komplette Bedeutung der Inschrift nur denjenigen, die (a) auch lesen (und schreiben) konnten, (b) den Psalm in seiner Wirksamkeit auf der Basis des kurzen Textes zu identifizieren und (c) mit einem bestimmten Schuss an Vertrauen und Glauben für die Wirksamkeit und Effizienz des Psalms und der anderen Elemente ausgestattet waren. Demnach könnte man hier auch von Outsidern sprechen, also jenen, die entsprechend (a), (b) und (c) nicht vorankommen und denen sich das Amulett insgesamt oder in Details nicht erschließt, und von Insidern, jenen Menschen, für die (a), (b) und/oder (c) in irgendeiner Art und Weise zutreffen. Auf alle Fälle war das Amulett leicht transportabel und damit immer präsent, wenn es das auch sein sollte. Über die Sichtbarkeit entschied der Träger/die Trägerin dadurch, dass es unter der Kleidung oder deutlich sichtbar getragen wurde.

35 Hierzu Mayser 1970², 60-65; Gignac 1976, 189-190. 


\subsection{Ein Armband im British Museum London}

Offensichtlich existierte eine Kategorie an Armbändern, die aufgrund diverser Charakteristika zusammengehörten. Nach meiner eigenen Zählung umfasst diese Gruppe vierunddreißig Objekte, von denen dreißig Text aus Psalm 90 ${ }^{\mathrm{LXX}}$ überliefern. ${ }^{36}$ Obgleich die Armbänder sich individuell sehr wohl unterscheiden, gerade was die künstlerische Gestaltung, Elemente des ikonographischen Programms und textliche Elemente angeht, sind es mehr Kriterien, welche die einzelnen Armbänder zu einer eigenen Kategorie miteinander verbinden. ${ }^{37}$ Die Armbänder stammen wahrscheinlich aus der Zeit zwischen der Mitte des sechsten und des siebten Jahrhunderts. Die meisten von ihnen wurden in Ägypten und in Syrien wie Palästina gefunden. Auch scheinen sie alle einem gemeinsamen Ursprungsmodell entsprungen zu sein bzw. sich aus zwei unabhängig voneinander entstandenen Ursprüngen entwickelt zu haben, so dass sich zwei regionale Untergruppen gebildet haben können, die sich jedoch nicht wesentlich unterscheiden lassen. Als Material fanden Silber, Bronze bzw. Kupferlegierung oder einfach nur Eisen Verwendung. Die Armbänder wurden vorzugsweise um den Unterarm getragen, wohl paarweise und/oder um den linken und den rechten Unterarm, was der Durchmesser vieler Exemplare von 7,5 cm nahelegt. Andere konnten auch um den Oberarm getragen werden oder waren für dünne Handgelenke gefertigt bzw. für Kinder hergestellt, gerade wenn der Durchmesser gerade mal 4 cm beträgt. Viele der Armbänder bestanden offensichtlich aus fünf Medaillons, von denen ein leeres, also nicht bearbeitetes, zum Verbinden unter ein anderes Medaillon gelötet wurde. Dennoch gibt es auch Armbänder mit nur drei Medaillons mit einem zusätzlichen vierten, leeren. Wie schon hinsichtlich der Medaillons und Anhänger bemerkt, existierte ein bestimmtes Programm an textlichen (z. B. die Eĩ $\theta$ có -Formel, das trishagion und Anrufungen) und ikonographischen (z. B. die Verkündigung an Maria, die Geburt Jesu, die Verehrung durch die Weisen, die Dreifaltigkeit, der Reiterheilige, Taufe Jesu, Kreuzigung Jesu, Frauen am Grab, Chnubis-Schlange ${ }^{38}$, Schiffsheck [ä $\varphi \lambda \alpha \sigma \tau o v]^{39}$ oder weniger häufigere Darstellungen), aber auch zeichenhaften (z. B. der böse Blick) und symbolischen Elementen für die Ausgestaltung von Reif und Medaillons. Nur neun der Armbänder sind ohne Vers bzw. irgendein Wort aus Psalm 90 ${ }^{\mathrm{LXX}}$ (meist nur der Beginn von Psalm 90,1), vier weisen ausschließlich dekorative Elemente auf. Einige textliche und ikonographische Elemente verdeutlichen den synkretistischen Hintergrund mancher Objekte, entstammen sie doch einer Welt

36 Weiterführend Kraus 2005/2006, 114-27 und Tafeln 2-3, sowie Kraus 2009, 143-147. Des Weiteren Kraus 2005, 52-57; Kraus 2009, 141-143; Kraus 2013, 143.

37 Vikan 1991/1992, 34.

38 Für wertvolle Hinweise zu Chnubis/Chnumis und die Zusendung seiner im Publikationsprozess befindlichen Habilitationsarbeit mit relevanten Hinweisen hierzu danke ich Joachim Friedrich Quack, Ägyptologisches Institut, Heidelberg.

39 Hierzu Casson 1971, Abb. 114, 116, 119-121 u. ö. 
unterschiedlicher, miteinander vermengter religiöser Vorstellungen, wie sie auch in Ägypten und Syrien/Palästina vorlag (so vor allem Chnubis, der böse Blick, magische Zeichen und Symbole, das Schiffsheck).

Ein genauer Blick auf eines dieser Armbänder im British Museum in London, AF 255+289, soll die materiellen Aspekte sowie Präsenz und Sichtbarkeit dieser archäologischen Objekte vor Augen führen. ${ }^{40}$
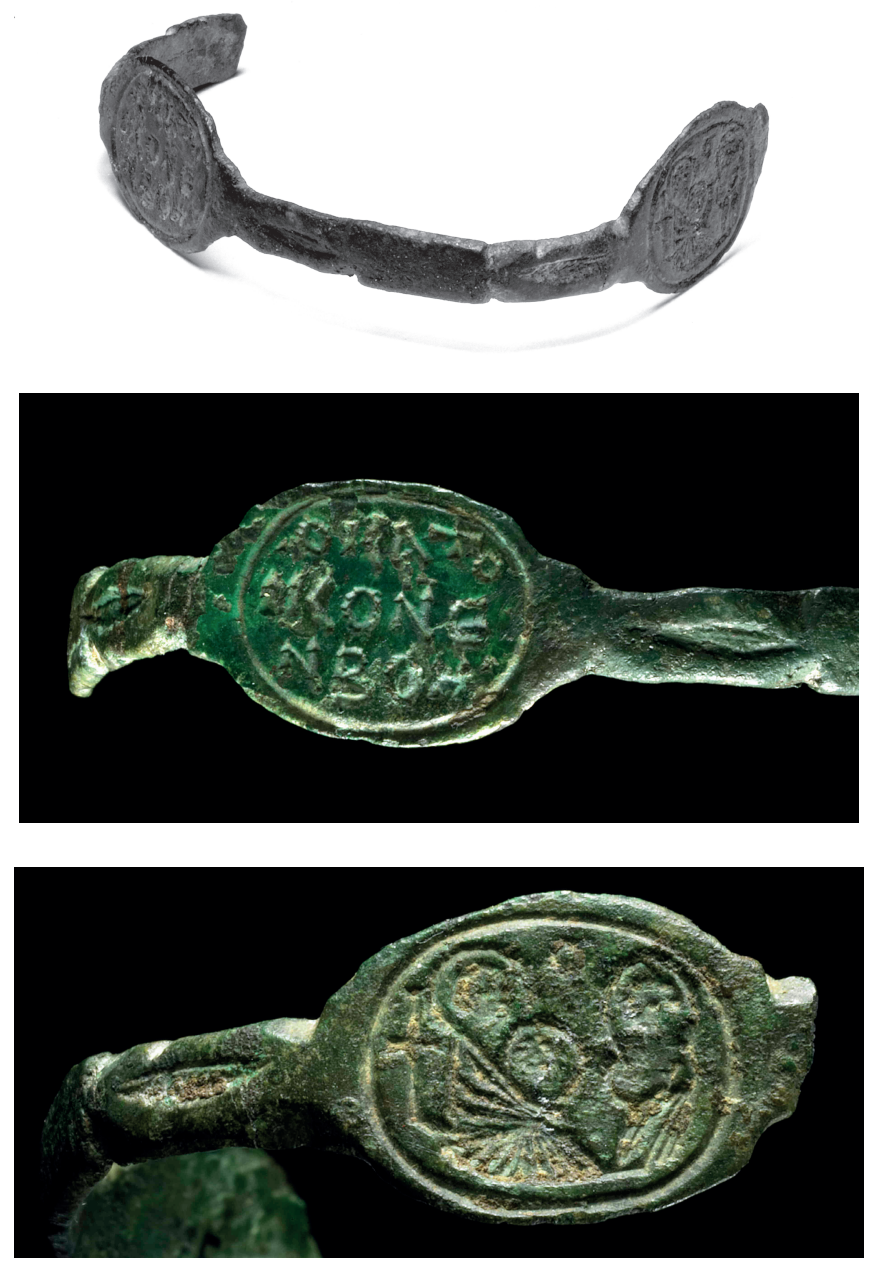

Abb. 3, 4 und 5: Fragmente eines Amulett-Armbands, London, British Museum, AF 255+289, mit dem Besuch der Weisen (AF 289) und dem Reiterheiligen (AF 255). (C) The Trustees of the British Museum.

40 Vgl. Kraus 2005/2006, 114-127. 
Das Armband ist nur mehr in zwei Fragmenten erhalten, die miteinander verbunden etwa die Hälfte des ursprünglichen Objekts ausmachen dürften. Immerhin liegen noch zwei Medaillons und zwei verbindende Teile des Reifs vor. Das Armband ist stark oxidiert (grünliche Korrosion des Kupfermaterials), aber textliche und ikonographische Elemente können rasch und leicht identifiziert werden. Das erste Medaillon

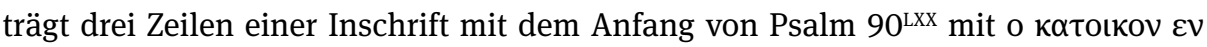
ßoך (OKATO | IKONE | NBOH). Das zweite zeigt eine sitzende Figur mit einer kleineren auf ihrem Schoß. Die sitzende Figur ist einer stehenden auf der anderen, der rechten Seite, zugewandt. Alle drei Personen haben einen Nimbus und in der Mitte zwischen den beiden größeren Figuren ist ein Stern auf Gesichtshöhe zu erkennen. Das ikonographische Repertoire der anderen Armbänder hilft dabei, die Personenkonstellation hier aufzulösen: Die Stofffalten der Bekleidung der sitzenden Person weisen auf eine Frau mit Kleid oder zumindest mit Rock hin. Maria mit dem Jesus-Kind auf ihrem Schoß nimmt die Huldigung eines der Weisen entgegen. Der Stern in der Mitte ist weiteres Charakteristikum der Szene, denn der Stern von Bethlehem wies den Weisen erst den Weg. Allerdings erscheint der Weise zunächst als Frauenfigur, da die Person in Brusthöhe einen weiblichen Brustansatz zu haben scheint. Dies jedoch kann eher das Geschenk des Weisen darstellen, das dieser auf seinen Händen in dieser Haltung präsentiert (hierzu Matthäus 2,1-12). Doch wo sind die anderen Weisen? Wie schon hinsichtlich Psalm 90 ${ }^{\mathrm{LXX}}$ vermerkt, genügten wenige Worte oder sogar nur der lapidare Anfang des Psalms, um den restlichen Text und die Wirksamkeit des ganzen Psalms zu vergegenwärtigen. Das Medaillon allein bietet nur begrenzten Raum, so dass ein Weiser genügt haben kann, um die Szene als solche darzustellen. Auf den beiden Resten des Reifs sind stilisierte Augen erkennbar, die mit diagonalen Linien durchgestrichen wurden. Dabei handelt es sich um eine typische Darstellung des bösen Blicks, der so unwirksam und entmachtet werden soll.

Damit kommt man der Funktion und angenommenen Wirksamkeit des Armbands als Schutzamulett näher. Die Armbänder, meist getragen an den Unterarmen und wohl paarweise, waren für andere sichtbar, Text und bildliche Darstellungen nach außen gerichtet. Übel und böse Mächte werden abgewehrt, wobei das Ganze anderen auch zu erkennen gegeben wird. Wiederum wird die Rezeption durch andere Personen, denen die Träger solcher Armbänder begegnen, davon abhängen, ob es sich um Insider oder Outsider handelt, denn die Bedeutung und Funktion der Bildelemente hängt letztlich von Wissen um sie ab, hinsichtlich des Textes kommt dann noch eine gewisse Fähigkeit des Lesens (und Schreibens) hinzu. Doch auch wer nicht genau um die Hintergründe von Bild und Text Bescheid wusste, dürfte durch die Praxis und im Alltag mit solchen Gegenständen konfrontiert worden und deshalb zumindest über einige Aspekte von Sinn und Zweck solcher Amulette informiert gewesen sein. 


\section{Mannigfaltige und variationsreiche Bezeugung von Psalm 90 ${ }^{\mathrm{Lx}}$ - Zusammenfassung}

Die aufgezeigte breite Bezeugung des Psalmtextes auf unterschiedlichen Materialien in verschiedener Mobilität, Größe und Ausgestaltung, stellt ein einzigartiges Phänomen dar: Sicherlich gibt es das eine oder andere auch für andere biblische Texte, die gerade für spezifische Zwecke populär und deren diesbezügliche Verwendung verbreitet war. Die Variationsbreite und die Häufigkeit erhaltener archäologischer Objekte mit Psalm 90 ${ }^{\mathrm{Lxx}}$ ist allerdings von anderen unerreicht. Dabei spielen für den vorgelegten Zusammenhang stets konkreter Verwendungskontext und Verwendungszweck eine determinierende Rolle für die Ausgestaltung, d. h. die ,künstlerische` Ausfertigung der Buchstaben bzw. der Verse, bzw. zumeist nur der beiden Anfangsworte (und diese nicht immer vollständig) und die Kompilation mit anderen Elementen. Und natürlich ist auch das verwendete Material wichtig, das nicht zuletzt etwa bei den Armbändern, Medaillons und Anhängern auch materiell und in seiner Ausformung klare Grenzen für den Umfang des dargebotenen Texts darstellt.

Türstürze und Sarkophage aus Steinmaterial etwa richten ihre Inschriften/Aufschriften an die Menschen, die auf ihrem Weg durch sie hindurchgehen oder an ihnen vorbeikommen. Die Sarkophage umfassen dadurch aber genauso die in ihnen Begrabenen, so dass sich die Inschriften auch an diese wenden. Natürlich sind sie aber ortsgebunden, so dass sich die apotropäische Funktion entsprechend anders darstellt als bei Papyri, die gefaltet und in Behältnissen mitgetragen, mobile Objekte darstellen und mit ihren Trägern unterwegs sind. Ihre Funktion ist damit deutlicher personengebunden, da ihre Präsenz mitentscheidend ist. In Grabkammern wiederum verschließen sich den Vorbeikommenden die Relevanz, Ausgestaltung und Wirksamkeit der Inschriften zunächst, vielmehr sogar ihre bloße Existenz. Textliches und Bildhaftes richten sich vielmehr an die Verstorbenen und möglicherweise auch die Sphäre der anderen Welt, genauer gesagt umrahmen sie die Toten, hüllen sie ein gegen alles Übel und schützen sie so.

Die Frage nach Sichtbarkeit, Präsenz, Mobilität aber auch Transportfähigkeit ist besonders relevant in Bezug auf Medaillons/Anhänger und Armbänder, wie sie anhand von zwei konkreten Beispielen auch visuell dargestellt wurde. Beide Objekte begleiten ihre Träger auf Schritt und Tritt, sofern sie nicht von diesen abgelegt werden, sind also stets dort, wo sich die Träger auch aufhalten. Text und Ikonographisches sind nach außen gerichtet und können - bei entsprechender Nähe zu den Trägern gesehen. erkannt und in ihrer Bedeutung erschlossen werden, was so auf gefaltete bzw. zusammengerollte und in Kapseln verwahrte Papyri nicht zutrifft. Also handelt es sich ansonsten um Nichts, das versteckt oder verborgen werden müsse, sei es, dass die Wehr gegen Böses und Übel unangemessen und inakzeptabel war, oder dass für die Wirksamkeit die Elemente zu den Trägern hin bzw. verborgen gehalten werden mussten. Übelabwehr findet also für die Träger immer und überall statt und ist eine 
auch von anderen offen wahrnehmbare Realität. Natürlich sind Insider in der Lage, Inhalt und Bedeutung von Text und Bild zu erschließen, ermächtigt durch ihr Wissen und ihre Erfahrung. Outsider dagegen können nicht alle Details erschließen, wenngleich davon auszugehen ist, dass das bloße Tragen von Medaillons/Anhängern und Armbändern möglicherweise zwar Schmuckcharakter hatte, vielmehr aber zu einem bestimmten (apotropäischen) Zweck erfolgte. Zumindest soviel dürfte auch Outsidern bekannt gewesen sein. Nach außen gerichtet also bannten Text und Bildhaftes alle Formen von Bösem und schützten die Träger solcher Amulette vor Übel, Krankheiten und Katastrophen, jedenfalls in der Vorstellung der Träger selbst.

\section{Literaturverzeichnis}

Breed, Brennan (2014), „Reception of the Psalms: The Example of Psalm 91“, in: William P. Brown (Hg.), The Oxford Handbook of the Psalms, Oxford, 297-310.

Casson, Lionel (1971), Ships and Seamanship in the Ancient World, Princeton.

Copeland, Gloria (2012), Your Promise of Protection: The Power of the 91st Psalm, Tulsa.

Demirer, Ünal (2013), Kibyra Metal Buluntuları - Metal Finds of Kibyra (Dissertation at Akdeniz Universitity, Ankara).

Demirer, Ünal/Kraus, Thomas J. (2015), „Ein Bronze-Amulett aus Kibyra mit Reiterheiligem und griechischem Psalm 90,1“, in: Zeitschrift für Papyrologie und Epigraphik 195, 58-62.

Felle, Antonio Enrico (2006), Biblio Epigraphica: La Sacra Scriptura nella Documentazione Epigrafica dell'Orbis Christianus Antiquus (III-VIII Secolo) (Inscriptiones Christianae Italiae Subsidia 5), Bari.

Foskolou, Vicky A. (2014), „The Magic of the Written Word: The Evidence of Inscriptions on Byzantine Magical Amulets“, in: Deltion of the Christian Archaeological Society 35, 329-348.

Gignac, F.T. (1976), Grammar of the Greek Papyri of the Roman and Byzantine Periods, Vol. 1: Phonology (Testi e documenti per lo studio dell'antichità 60), Mailand.

Hugger, Pirmin (1971), Jahwe meine Zuflucht. Gestalt und Theologie des 91. Psalms (Münsterschwarzacher Studien, Reihe theologischer und geisteswissenschaftlicher Untersuchungen 13), Münsterschwarzach.

Kraus, Thomas J. (2005), „Septuaginta-Psalm 90 in apotropäischer Verwendung: Vorüberlegungen für eine kritische Edition und (bisheriges) Datenmaterial“, in: Biblische Notizen 125, 39-73.

Kraus, Thomas J. (2005/2006), „Fragmente eines Amulett-Armbands im British Museum (London) mit Septuaginta-Psalm 90 und der Huldigung der Magier“, in: Jahrbuch für Antike und Christentum 48/49, 114-27 und Tafeln 2-3.

Kraus, Thomas J. (2006a), „Manuscripts with the Lord's Prayer - They are more than simply Witnesses to that Text itself“, in: Thomas J. Kraus u. Tobias Nicklas (Hgg.), New Testament Manuscripts. Their Texts and Their World (Texts and Editions for New Testament Study 2), Leiden/Boston, 227-266.

Kraus, Thomas J. (2006b), „,Der Herr wird deinen Eingang und deinen Ausgang bewahren“: Über Herkunft und Fortleben von LXX Psalm cxx 8a“, in: Vetus Testamentum 56, 58-75.

Kraus, Thomas J. (2007), „,Parchment or Papyrus?": Some Remarks about the Significance of Writing Material when Assessing Manuscripts'“, in: Thomas J. Kraus (Hg.), Ad Fontes: Original Manuscripts and Their Significance for Studying Early Christianity (Texts and Editions for New Testament Study 3), Leiden/Boston, 13-24. 


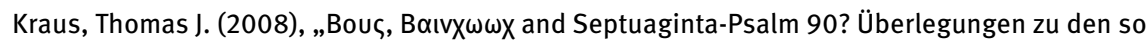
genannten ,Bous'-Amuletten und dem beliebtesten Bibeltext für apotropäische Zwecke“, in: Zeitschrift für antikes Christentum 11, 479-491.

Kraus, Thomas J. (2009), „,He that Dwelleth in the Help of the Highest': Septuagint Psalm 90 and the Iconographic Programme on Byzantine Armbands", in: Craig A. Evans u. H. Daniel Zacharias (Hgg.), Jewish and Christian Scripture as Artifact and Canon (Studies in Scripture in Early Judaism and Christianity 13), London/New York, 137-147.

Kraus, Thomas J. (2013), „Der lukianische bzw. antiochenische Text der Psalmen in Papyri und Inschriften. Eine Suche nach der Stecknadel im Heuhaufen?“, in: Siegfried Kreuzer und Marcus Sigismund (Hgg.), Der Antiochenische Text der Septuaginta in seiner Bezeugung und seiner Bedeutung (De Septuaginta Investigationes 4), Göttingen, 133-162.

Kraus, Thomas J. (2014), „,Außertextliche“ Rezeption von LXX-Psalm 90 - ,Lebensgeschichte und Lebendigkeit eines Psalms“, in: Wolfgang Kraus, Siegfried Kreuzer, Martin Meiser u. Marcus Sigismund (Hgg.), Die Septuaginta: Text, Wirkung, Rezeption (4. Internationale Fachtagung veranstaltet von Septuaginta Deutsch (LXX.D), Wuppertal 19.-22. Juli 2012), (Wissenschaftliche Untersuchungen zum Neuen Testament 325), Tübingen, 825-838.

La'da, Csaba/Papathomas, Amphilochios (2004), „A Greek Papyrus Amulet from the Duke Collection with Biblical Excerpts“, in: Bulletin of the American Society of Papyrologists 41, 93-113.

Mayser, Edwin (1970²), Grammatik der griechischen Papyri aus der Ptolemäerzeit. Bd. 1: Laut- und Wortlehre, 1. Teil: Einleitung und Lautlehre, Berlin.

Mills, Owing (2013), „A Sinner Holds Tight to Faith and a Second Chance“, in: The New York Times (January 11), http://www.nytimes.com, Suchfunktion [search] (letzter Zugriff 30.07.2015).

Nachtergael, G. (2002), „Une amulette chrétienne du Musée des Beaux-Arts de Dijon“, in: Ricerche di egittologia e di antichità copte 4, 93-101.

Pasternak, Boris (1992), Doktor Shiwago, übers. von T. Reschke, Berlin.

Prentice, William K. (1908), Greek and Latin Inscriptions. Part III of the Publications of an American Archaeological Expedition to Syria in 1899-1900, New York.

Rebiger, Bill (2003), Sefer Shimmush Tehillim - Buch vom magischen Gebrauch der Psalmen. Edition, Übersetzung und Kommentar (Texts and Studies in Ancient Judaism 137), Tübingen.

Ruth, Peggy Joyce (2007), Psalm 91: God's Shield of Protection, Lake Mary/Florida.

Sanzo, Joseph E. (2014), Scriptural Incipits on Amulets from Late Antique Egypt (Studien und Texte zu Antike und Christentum 84), Tübingen.

Schilken, Chuck (2013), „Ray Lewis’ postgame T-shirt brings attention to Psalm 91“, Los Angeles Times (January 07), http://articles.latimes.com/2013/jan/07/sports/la-sp-sn-ray-lewispsalm-91-20130107 (letzter Zugriff 30.07.2015).

Vikan, Gary (1991/1992), „Two Byzantine amuletic armbands and the group to which they belong“, in: The Journal of the Walters Art Gallery 49/50, 33-51 (= idem (2003), Sacred images and sacred power in Byzantium (Variorum Collected Studies Series), Aldershot/Burlington, article $\mathrm{X})$. 
